\title{
Development and validation of a risk prediction score for severe acute pancreatitis
}

\author{
Wandong Hong ${ }^{1 *}+$ CD, Keith D. Lillemoe ${ }^{2}$, Shuang Pan ${ }^{3}$, Vincent Zimmer ${ }^{4,5{ }^{\dagger}}$, Evangelos Kontopantelis ${ }^{6,7 \dagger}$,
} Simon Stock ${ }^{8}$, Maddalena Zippi ${ }^{9+}$, Chao Wang ${ }^{10+}$ and Mengtao Zhou ${ }^{11^{*}}$

\begin{abstract}
Introduction: The available prognostic scoring systems for severe acute pancreatitis (SAP) have limitations that restrict their clinical value. The aim of this study was to develop a simple model (score) that could rapidly identify those at risk for SAP.
\end{abstract}

Methods: We derived a risk model using a retrospective cohort of 700 patients by logistic regression and bootstrapping methods. The discriminative power of the risk model was assessed by calculating the area under the receiver operating characteristic curves (AUC). The classification and regression tree (CART) analysis was used to create risk categories. The model was internally validated by a tenfold cross-validation and externally validated in a separate prospective cohort of 194 patients.

Results: The incidence of SAP was $9.7 \%$ in the derivation cohort and $9.3 \%$ in the validation cohort. A prognostic score (We denoted it as the SABP score), ranging from 0 to 10, consisting of systemic inflammatory response syndrome, serum albumin, blood urea nitrogen and pleural effusion, was developed by logistic regression and bootstrapping analysis. Patients could be divided into three risk categories according to total SABP score based on CART analysis. The mean probability of developing SAP was $1.9 \%, 12.8 \%$ and $41.6 \%$ in patients with low (0-3), moderate (4-6) and high (7-10) SABP score, respectively. The AUCs of prognostic score in tenfold cross-validation was 0.873 and 0.872 in the external validation.

Conclusion: Our risk prediction score may assist physicians in predicting the development of SAP.

Keywords: Prediction, Acute pancreatitis, Severity, Risk factor, Score

\section{Introduction}

Though most patients with acute pancreatitis (AP) suffer from a mild and self-limiting form with a benign clinical course $[1,2]$. Approximately $10-20 \%$ of all cases present

\footnotetext{
*Correspondence: xhnk-hwd@163.com; studyzhoumengtao@sina.com tWandong Hong, Vincent Zimmer, Evangelos Kontopantelis, Maddalena Zippi and Chao Wang contributed equally to this work

1 Department of Gastroenterology and Hepatology, The First Affiliated Hospital of Wenzhou Medical University, Nanbaixiang, Ouhai District, Wenzhou 325000, Zhejiang, People's Republic of China

${ }^{11}$ Department of Surgery, Key Laboratory of Diagnosis and Treatment of Severe Hepato-Pancreatic Diseases of Zhejiang Province, The First Affiliated Hospital of Wenzhou Medical University, Wenzhou, Zhejiang, People's Republic of China

Full list of author information is available at the end of the article
}

with severe acute pancreatitis (SAP), which is associated with a significant risk of mortality $[3,4]$.

Early identification of high-risk patients on admission may help physicians to select those patients who would benefit the most from close surveillance, or aggressive intervention [5]. Firstly, early identification of patients who have a high probability of developing SAP in the emergency room may benefit from close surveillance, aggressive critical care and early treatment $[5,6]$. Early aggressive intravenous hydration is most beneficial in the first $12-24 \mathrm{~h}$ and may have little benefit beyond this time [2, 7]. Early fluid resuscitation may improve microcirculation of the pancreas and provide hemodynamic support, which results in reduced incidence

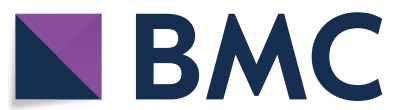

(c) The Author(s) 2019. This article is distributed under the terms of the Creative Commons Attribution 4.0 International License (http:// creativecommons.org/licenses/by/4.0/), which permits unrestricted use, distribution, and reproduction in any medium, provided you give appropriate credit to the original author(s) and the source, provide a link to the Creative Commons license, and indicate if changes were made. The Creative Commons Public Domain Dedication waiver (http://creativecommons.org/publicdomain/zero/1.0/) applies to the data made available in this article, unless otherwise stated. 
of morbidity and mortality among patients with acute pancreatitis [8, 9]. Early endoscopic retrograde cholangiopancreatography (ERCP) is associated with fewer complications in predicted severe acute biliary pancreatitis [10]. Secondly, it was reported that patients in high-volume centers had a shorter length of stay, lower hospital charges, and lower mortality rates than do those in low-volume centers [11]. As a result, clinicians need to identify those patients who do not respond to early resuscitation or display SAP for possible transfer to specialist care or a pancreatitis centre if available $[2,6]$. Lastly, the ability to identify patients at risk of SAP early in the disease course also helps in designing mechanistic studies or clinical trials for targeted intervention [3].

Many clinical scoring systems have been developed, such as the Bedside index of severity in acute pancreatitis (BISAP) [12],chronic health evaluation (APACHEII) score, and modified Glasgow score, Japanese severity score (JSS) [13], and the Harmless acute pancreatitis score (HAPS) [14]. However, these existing scoring systems were primarily derived for prediction of severe disease based on the Atlanta criteria or mortality but not for SAP defined by recent revised international guidelines on acute pancreatitis $[3,6]$. Although individual predictors, such as admission hematocrit $(\geq 44 \%)$ or blood urea nitrogen (BUN) at $24 \mathrm{~h}$, are easy to use in practice, they lack high sensitivity or specificity [15].

Therefore, the aim of this work was to develop and validate a simple risk score for the early prediction of SAP.

\section{Patients and methods}

\section{Inclusion and exclusion criteria}

Patients with acute pancreatitis admitted to the First Affiliated Hospital of Wenzhou Medical University (Wenzhou City, Zhejiang Province, China) within $72 \mathrm{~h}$ of symptom onset from January 2012 to December 2015 were retrospectively included in the derivation cohort [16]. Thereafter, patients with acute pancreatitis from January 2016 to December 2016 in the First Affiliated Hospital of Soochow University (Suzhou City, Jiangsu Province, China) were prospectively included in the validation cohort. Acute pancreatitis was defined as previously described $[1,6]$. According to the revised Atlanta classification, SAP is characterized by single or multiple organ failure (respiratory, cardiovascular, renal) that persists for $>48 \mathrm{~h}[2,6]$.

Exclusion criteria were [16]; patients that had developed organ failure before data collection, previous pancreatic surgery, recurrent or not first-time pancreatitis, pancreatitis due to endoscopic retrograde cholangiopancreatography (ERCP) or trauma, chronic pancreatitis, pancreatic cancer, pleural effusions both preceding the development of AP and as the result of concomitant diseases (e.g., pneumonia, chronic heart failure), chronic renal disease, patients with albumin infusion before data collection in our hospital, hypoalbuminemia due to malnutrition, albuminuria, hepatitis, liver cirrhosis.

\section{Data collection}

Age, gender, body mass index (BMI), time from symptom onset to admission and biochemical parameters were recorded within $12 \mathrm{~h}$ of hospitalization, except for serum albumin levels which were assayed within the first $24 \mathrm{~h}$ [16]. All patients underwent abdominal computed tomography $(\mathrm{CT})$ scan within $6 \mathrm{~h}$ of admission and the presence of a pleural effusion was recorded. Data for every variable of systemic inflammatory response syndrome (SIRS), BISAP, APACHE II, HAPS, Glasgow and JSS scores were collected if available and were calculated as described by Wu et al. [12] and Mounzer et al. [3].

\section{Statistical analysis}

Categorical variables were described using frequencies and proportions and compared using $\mathrm{X}^{2}$ tests. Continuous values were expressed using mean \pm standard deviation (SD), or median and interquartile range (IQR) and compared using Student's $t$ test or the nonparametric Mann-Whitney test. Linear trend of categorical and continuous variables was tested using a Royston extension of the Cochran-Armitage test [17] and a non-parametric Wilcoxon rank sum test [18], respectively.

For easier application to a risk score model, when performing multivariate logistic regression analysis, most continuous variables were converted to categories based on published data as follows: advanced age ( $\geq 60$ years) [12], body mass index (BMI) $(\geq 30)$ [19], SIRS (yes vs. no) [12], hematocrit $(\geq 44 \%)$ [15], platelet count $(\leq 100,000 /$ $\mathrm{mm}^{3}$ ) [20], prothrombin time (PT $\geq 18 \mathrm{~s}$ ) [21], total bilirubin $(\geq 2 \mathrm{mg} / \mathrm{dL}$ ), alanine aminotransferase (ALT $>50$ $\mathrm{U} / \mathrm{L}$ ), aspartate aminotransferase (AST $>45 \mathrm{U} / \mathrm{L}$ ) [22], glucose $(\geq 150 \mathrm{mg} / \mathrm{dL})$ [19], blood urea nitrogen (BUN) ( $>25 \mathrm{mg} / \mathrm{dL}$ ) [12] and pleural effusion (yes vs. no) [5].

Candidate predictors with $\mathrm{P}<0.20$ in univariate analyses were included a multivariate logistic regression. In addition, a backward stepwise bootstrap regression model, in which 1000 random samples patients were generated with replacement, was also performed to investigate the relative importance of each variable included in our model [23]. Frequencies of occurrence of each covariate in the final model were noted; if predictors occurred in $90 \%$ or more of the bootstrap models, they were retained in the final multivariate model [24]. Beta regression coefficients and odds ratios (OR) were calculated with $95 \%$ confidence intervals $(\mathrm{CI})$. The multivariate 
regression coefficients of the predictive factors were used to assign integer points for the prediction score [25, 26]. Individual risk estimates were based on the sum of weighted scores for each variable.

The discriminative power of the prediction score was assessed by calculating the area under the receiver operating characteristic (ROC) curves (AUC) [27]. All variables were used as continuous variables when calculating AUC. A predictor with an AUC above 0.7 was considered to be useful, while an AUC between 0.8 and 0.9 indicated good diagnostic accuracy [28].

The model was internally validated using tenfold cross-validation $[29,30]$. When performing tenfold cross-validation, we first randomly divided all data into ten equal-sized subsamples. The aim is to use nine subsamples for training and the remaining one for testing, over all possible permutations. Through the cross-validation process, the analysis is then repeated ten times (folds), with each of the ten subsamples used exactly once as the validation data [30]. The AUC is calculated for each of the 10 analyses, using only the respective test data, and these 10 AUC statistics are then further aggregated into means, standard deviation (through which 95\% confidence intervals are calculated), medians, etc. [29]. The classification and regression tree (CART) analysis was used to create risk categories according to total prediction score [5]. When performing CART analysis, impurity function was used for splitting and cut-off points for continuous variables which were generated automatically based on statistical cost assumptions [5]. Calibration of the risk score reflecting the link between predicted and observed risk, was evaluated by the Hosmer-Lemeshow goodness of fit test [31].

A $P$ value $<0.05$ was considered statistically significant for all analyses. Data were analyzed using the STATA version 12 and R 3.5.1 statistical software.

\section{Results}

\section{Characteristics of the investigated population}

Distributions for demographic and clinical features between the two study populations are depicted in Table 1. There were 700 and 194 patients enrolled in the derivation cohort and validation cohort respectively. Biliary cause was the most common etiology in $42.7 \%$ of patients in the derivation cohort and $38.7 \%$ of patients in the validation cohort. The incidence of SAP was 9.7\% $(68 / 700)$ and $9.3 \%(18 / 194)$ in the derivation cohort and validation cohort respectively.

\section{Univariate and multivariate analysis in the derivation cohort}

Sixteen variables considered relevant to the presence of SAP were tested using univariate analysis (Additional

\begin{tabular}{|c|c|c|}
\hline Variable & $\begin{array}{l}\text { Derivation } \\
\text { cohort }(n=700)\end{array}$ & $\begin{array}{l}\text { Validation } \\
\text { cohort } \\
(n=194)\end{array}$ \\
\hline Age, years (IQR) & $48(37-63)$ & $49(38-61)$ \\
\hline Male sex, N (\%) & $435(62.1)$ & $127(65.5)$ \\
\hline Duration of symptoms, days & $1.83 \pm 0.79$ & $1.67 \pm 0.78$ \\
\hline SIRS, N (\%) & $272(38.9)$ & $82(42.3)$ \\
\hline \multicolumn{3}{|l|}{ Etiology } \\
\hline Biliary, N (\%) & $299(42.7)$ & $74(38.1)$ \\
\hline Alcohol, N (\%) & $96(13.7)$ & $12(6.2)$ \\
\hline Hypertriglyceridemia, N (\%) & $37(5.3)$ & $31(16.0)$ \\
\hline Idiopathic, N (\%) & $246(35.1)$ & $73(37.6)$ \\
\hline Other, N (\%) & $22(3.1)$ & $4(2)$ \\
\hline \multicolumn{3}{|l|}{ Laboratory findings } \\
\hline Hematocrit & $0.42(0.38-0.46)$ & $0.43(0.39-0.46)$ \\
\hline Platelets $\left(10^{9} / \mathrm{L}\right)$ & $197(158-233)$ & $206(169-248)$ \\
\hline Prothrombin time, s (IQR) & $13.8(13.1-14.6)$ & $13.7(13.2-14.5)$ \\
\hline Albumin, g/L (IQR) & $36.2(32.9-39.7)$ & $36.6(32.7-39.7)$ \\
\hline Bilirubin, mg/dL (IQR) & $1.16(0.79-1.81)$ & $1.11(0.76-1.75)$ \\
\hline ALT, U/L (IQR) & $40(19-107)$ & $33(18-91)$ \\
\hline AST, U/L (IQR) & $36(21-85)$ & $33(23-73)$ \\
\hline Glucose, mg/dL (IQR) & $144(117-193)$ & $139(112-191)$ \\
\hline BUN, mg/dL (IQR) & $13.4(10.4-17.4)$ & $14.0(10.6-17.9)$ \\
\hline Pleural effusion, N (\%) & $135(19.4)$ & $83(42.8)$ \\
\hline \multicolumn{3}{|l|}{ Clinical outcomes } \\
\hline Patients with SAP, N (\%) & $68(9.7)$ & $18(9.3)$ \\
\hline \multicolumn{3}{|l|}{ Number of POF, N (\%) } \\
\hline One organ failure & 38/68 (55.9) & $7 / 18(38.9)$ \\
\hline Two organ failure & 19/68 (27.9) & 6/18 (33.3) \\
\hline Three organ failure & $11 / 68(16.2)$ & $5 / 18(27.8)$ \\
\hline \multicolumn{3}{|l|}{ Type of POF } \\
\hline Respiratory failure, N (\%) & $56 / 68(82.4)$ & 16/18 (88.9) \\
\hline Renal failure, N (\%) & 24/68 (35.3) & 10/18 (55.6) \\
\hline Cardiovascular failure, N (\%) & 29/68 (42.7) & $8 / 18(44.4)$ \\
\hline Length of hospital stay, days (IQR) & $10(7-9)$ & $11(7-17)$ \\
\hline Hospital mortality, N (\%) & $11(1.6)$ & $2(1.0)$ \\
\hline
\end{tabular}

Data were mean \pm standard deviation, or numbers and percentages, or median (25th-75th percentile), as appropriate

$\mathrm{N}$, number; IQR, interquartile range; BMI, body mass index; SIRS, systemic inflammatory response syndrome; ALT, alanine aminotransferase; AST, aspartate aminotransferase; BUN, blood urea nitrogen; SAP, severe acute pancreatitis; POF, persistent organ failure

file 1: Table S1). Age, BMI, alcohol etiology, SIRS, hematocrit, platelet count, prothrombin time, albumin, AST, glucose, BUN and pleural effusion were identified as candidate predictors $(\mathrm{P}<0.20)$ of SAP in univariate analysis. When using these potential predictors (except albumin) as categories, all remained independently associated with SAP in multivariate logistic regression analysis: SIRS (odds ratios (OR) 2.98; 95\% confidence 
interval (CI) 1.47-6.04; $\mathrm{P}=0.003$ ), hematocrit (OR 2.24; 95\% CI 1.13-4.46; $\mathrm{P}=0.021$ ), albumin (OR 0.51; 95\% CI 0.36-0.73; P<0.001), AST (OR 2.18; 95\% CI $1.13-4.19 ; \mathrm{P}=0.02)$, serum glucose (OR 2.27; 95\% CI 1.12-4.61; $\mathrm{P}=0.023$ ), BUN (OR 4.58; 95\% CI 2.16-9.70; $\mathrm{P}<0.001$ ) and pleural effusion (OR 4.68; 95\% CI 2.429.05; $\mathrm{P}<0.001)$.

\section{Bootstrap analysis of potential predictors and development of prediction score in the derivation cohort}

The bootstrap analysis revealed that, out of twelve potential predictors, SIRS, albumin, BUN and pleural effusion were reproducibly selected in more than $90 \%$. Therefore, these four variables were kept in the final model for the development of the prediction score. The final logistic regression function was: $\log ($ odds of SAP $)=0.55+1.02$ (SIRS)-0.63 (albumin) + 1.76 (BUN) + 1.66 (pleural effusion). The logistic regression coefficients and 95\% CI, as well as the allocation of scoring points for each predictive factor based on the regression coefficients, are given in Table 2. We denoted it as the SABP (SIRS, albumin, BUN and pleural effusion) score. The total prediction score ranges between 0 and 10 with a high score indicating high risk of developing SAP.

\section{Discrimination and internal cross-validation of prediction score in the derivation cohort}

Based on ROC curve analysis in the derivation cohort (Fig. 1), the SABP score achieved higher AUC than other prediction scoring systems. The AUCs for SABP, BISAP, APACHE II, HAPS, Glasgow score, JSS score and CRP in the prediction of SAP were $0.875 \pm 0.023,0.834 \pm 0.024$,

Table 2 Point allocation for predictors of severe acute pancreatitis based on regression coefficients

\begin{tabular}{lcl}
\hline Predictive factor & Regression coefficient & Score assigned \\
\hline SIRS (yes vs. no) & $1.02(0.39-1.66)$ & 2 \\
Yes & & 0 \\
No & & \\
Albumin (5 g/L increments) & $-0.63(-0.95,-0.31)$ & \\
$\geq 35$ & & 1 \\
$30-34.9$ & $1.76(1.06-2.46)$ & 2 \\
$<30$ & & 3 \\
BUN (>25 mg/dL & \\
Vs. $\leq 25 \mathrm{mg} / \mathrm{dL})$ & 0 \\
$>25 \mathrm{mg} / \mathrm{dL}$ & \\
$\leq 25 \mathrm{mg} / \mathrm{dL}$ & $1.66(1.04-2.27)$ & 0 \\
Pleural effusion & & 3 \\
No & \\
Yes & \\
\hline
\end{tabular}

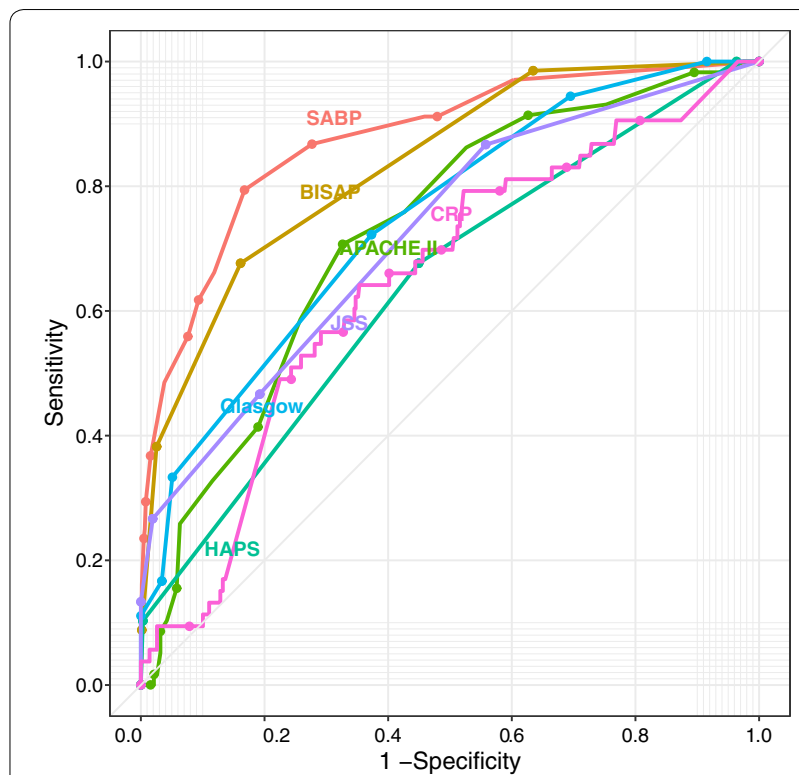

Fig. 1 Receiver operating characteristic (ROC) curve for SABP, BISAP, APACHE II (complete data available in 248 patients), HAPS, Glasgow score (complete data available in 77 patients), JSS score (complete data available in 67 patients) and CRP (complete data available in 551 patients) in derivation cohort. AUC, area under the curve of the receiver operating characteristic curve

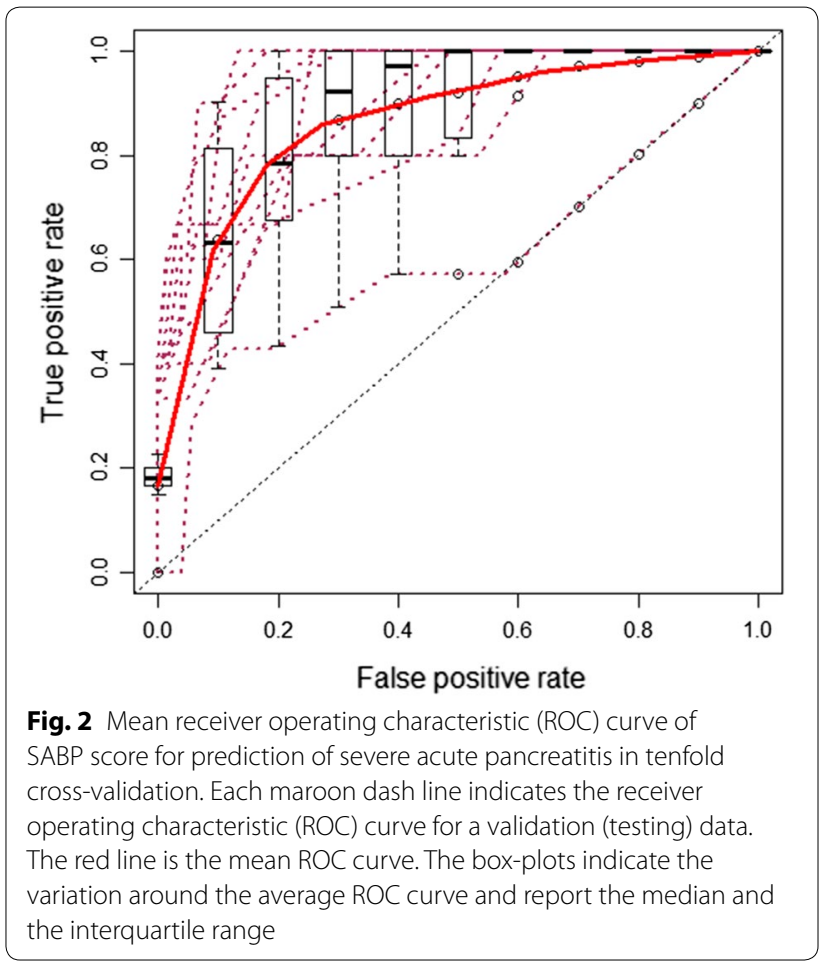

$0.725 \pm 0.037,0.642 \pm 0.032,0.746 \pm 0.063,0.724 \pm 0.073$ and $0.646 \pm 0.039$, respectively. 


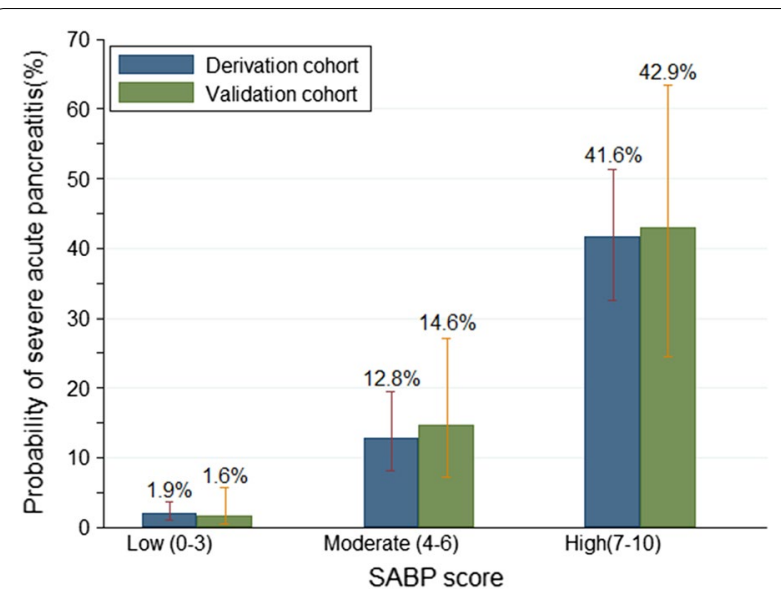

Fig. 3 Patients stratified by the SABP score in the derivation cohort and validation cohort according to classification and regression tree (CART) analysis

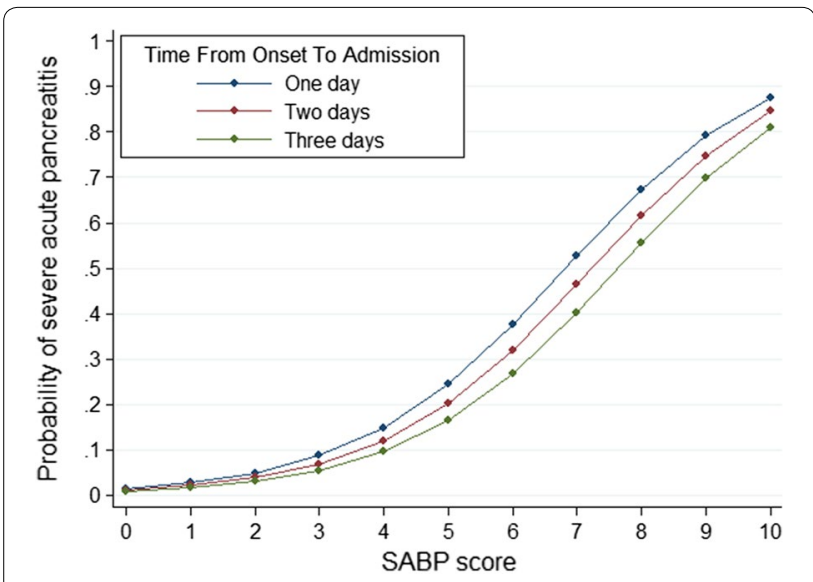

Fig. 4 Predicted probability of severe acute pancreatitis in acute pancreatitis over the SABP score from 0 to 10 for different onset-to-admission time

The mean ROC curve of tenfold cross-validation of the SABP score is shown in Fig. 2, which gave an AUC of the 0.873 (95\% CI 0.822-0.924) indicating good discrimination for our model. The Hosmer-Lemeshow goodness of fit test of tenfold cross-validation did not reach statistical significance $(P=0.631)$ indicating a good match of predicted risk over observed risk.

\section{Application of prediction score in the derivation cohort} As shown in Fig. 3, based on CART analysis, patients with acute pancreatitis in the derivation cohort could be divided into three risk categories according to total prediction score: low SABP score (score: 0-3), moderate SABP score (score: 4-6) and high SABP score (score:
7-10). The mean observed probability of developing SAP were $1.9 \%$ (9/466), $12.8 \%(17 / 133)$ and $41.6 \%(42 / 101)$ in patients with low, moderate and high SABP score, respectively. This indicated that a higher SABP score was associated with an increased risk of SAP $\left(P_{\text {trend }}<0.001\right)$.

In clinical practice, the time period from onset of pain to hospital admission may play a role in the occurrence and/or severity of SIRS, hypoalbuminemia, pleural effusion and increased BUN [32-34]. Therefore, we computed the predicted probability of SAP over the SABP score from 0 to 10 for different onset-to-admission times (Fig. 4). As an example, using the prevalence of SAP in acute pancreatitis (9.7\% in the derivation cohort), a patient with acute pancreatitis admitted to hospital within 1 day after the onset of abdominal pain, with SIRS, a serum albumin level of $33 \mathrm{~g} / \mathrm{L}$, BUN level of $26 \mathrm{mg} /$ $\mathrm{dL}$ and no pleural effusion would generate a score of 6 points. This translates to a $37.5 \%$ probability of developing SAP. However, the probability of developing SAP would decrease to $26.6 \%$ for patients with an onset-toadmission time of 3 days with the same score (6 points).

\section{Performance of prediction score in the validation cohort}

Based on ROC curve analysis in the validation cohort (Fig. 5), the SABP score achieved higher AUC than other prediction scoring systems. The AUCs for SABP, BISAP, APACHE II, HAPS, Glasgow score, JSS score and CRP in the prediction of SAP were $0.8725 \pm 0.049,0.8259 \pm 0.0497$, $0.789 \pm 0.059,0.636 \pm 0.055, \quad 0.721 \pm 0.057,0.703 \pm 0.062$, $0.623 \pm 0.070$, respectively.

Using CART analysis, patients with acute pancreatitis in the validation cohort could still be divided into the same three risk categories according to total prediction

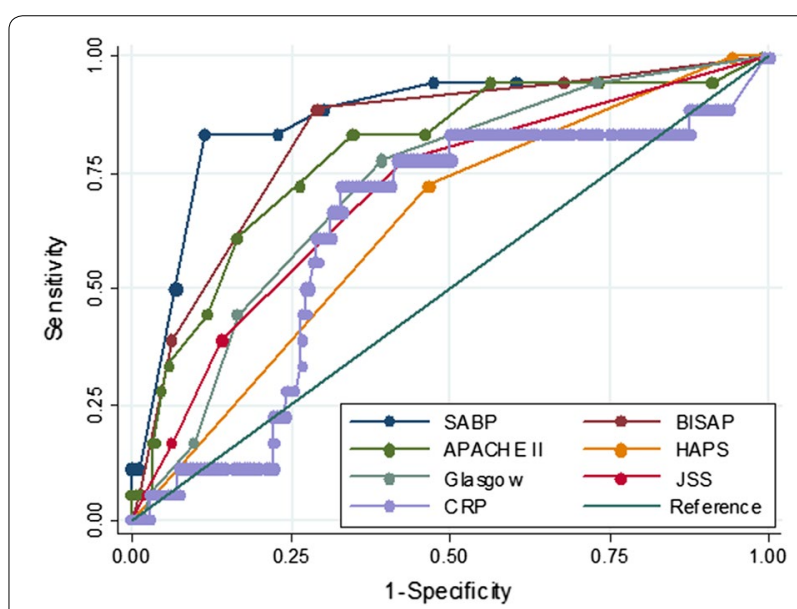

Fig. 5 Receiver operating characteristic (ROC) curve for SABP, BISAP, APACHE II, HAPS, Glasgow score, JSS score and CRP in validation. AUC, area under the curve of the receiver operating characteristic curve 
score) (Fig. 3). The mean observed probability of developing SAP was $1.6 \%(2 / 125), 14.6 \%(7 / 48)$ and $42.9 \%(9 / 21)$ in patients with low, moderate and high SABP score, respectively. This indicated that a higher SABP score was associated with an increased risk of SAP $\left(P_{\text {trend }}<0.001\right)$.

\section{Discussion}

The early extensive systemic release of proinflammatory cytokines, such as interleukin (IL)- 1 and IL-6 in patients with acute pancreatitis may give rise to SIRS [35, 36]. Mofidi et al. [35] found that persistent SIRS is associated with multi-organ dysfunction syndrome and death from acute pancreatitis. Singh et al. [36] suggested that patients with a higher number of SIRS criteria on the first day of hospitalization and persistent SIRS had an increased risk of SAP, as defined by persistent organ failure, pancreatic necrosis, need for intensive care unit, and death. Our data suggested that SIRS with an OR of 2.98 (95\% CI 1.47-6.04) was independently associated with SAP defined by the up-to-date revised Atlanta criteria.

Hypoalbuminemia may occur in patients with acute pancreatitis due to impaired liver synthesis, increased tissue catabolism and re-distribution from the intravascular to the interstitial space [16]. On the other hand, hypoalbuminemia can lead to the development of pulmonary edema and exacerbation of acute heart failure due to decreased colloid osmotic pressure [37]. Xue et al. [38] suggested that hypoalbuminemia in the early stage was associated with a high incidence of infection and mortality. Our data suggested that an increase of $5 \mathrm{~g} / \mathrm{L}$ serum albumin level was associated with a statistically significant $49 \%$ reduction in the odds of SAP (OR 0.51; 95\% CI 0.36-0.73).

A rise in the BUN level at admission in patients with acute pancreatitis may be secondary to pre-renal azotemia due to initial hypovolemia, a state of ongoing negative nitrogen balance related to increased protein catabolism induced by acute pancreatitis, and impairment of renal function $[39,40]$. Two large studies have reported that an elevated BUN level at admission is an independent risk factor for mortality in acute pancreatitis [12, 40]. Koutroumpakis et al. [15] indicated that a rise in BUN at $24 \mathrm{~h}$ outperforms other laboratory markers in predicting persistent organ failure and pancreatic necrosis in acute pancreatitis. Our results showed a positive association between an initial increased BUN level at admission and the development of SAP in acute pancreatitis.

Pleural effusion is often observed during acute pancreatitis. A possible explanation is that pancreatic duct disruption results in leakage of pancreatic secretions directly into the peritoneal cavity via the transdiaphragmatic lymphatic channels. Maringhini et al.
[41] found that the presence of pleural effusion was associated with an increased incidence of pancreatic pseudocyst in acute pancreatitis. Heller et al. [42] demonstrated a correlation between pleural effusion on chest radiograph and severity in accordance with the Atlanta criteria. The present study suggests that pleural effusion with an OR of 4.68 (95\% CI 2.42-9.05) was a strong individual predictor of SAP defined by the upto-date revised Atlanta criteria.

The application of the proposed SABP score is expected to change current clinical practice in the management of acute pancreatitis. Patients with high SABP scores may have much more pronounced risk factors, such as SIRS, pleural effusion, elevated BUN levels and low albumin, enhancing the risk of developing SAP (Figs. 3, 4). Therefore, in order to prevent occurrence of SAP, patients with high SABP scores should be monitored more carefully or even transferred to intensive care units (for example, for respiratory support for SIRS). These patients should also receive more active intravenous fluid therapy to correct intravascular volume depletion so as to decrease high BUN levels [19]. Additional interventions could be evaluated for relevance in this setting, and especially for high SABP scores. For example, it is well established that albumin infusion improves outcome of patients with septic shock [43], liver cirrhosis with hepatorenal syndrome [44] or spontaneous bacterial peritonitis [45]. Therefore, an interesting hypothesis would be whether the administration of albumin in patients with acute pancreatitis and hypoalbuminemia could decrease mortality or prevent development of SAP, since severe acute pancreatitis shares many features with sepsis syndrome and septic shock [46]. A future study could aim to evaluate the role of albumin replacement in the treatment of acute pancreatitis with hypoalbuminemia.

There is no consensus as to the best prognostic markers in acute pancreatitis in the literature. The APACHE II score requires the collection of a large number of parameters, which makes it clinically cumbersome so that APACHE II is seldom used in clinical practice [47, 48]. The HAPS was primarily developed for rapid initial identification of patients with a first attack of acute pancreatitis who do not require intensive care but not for prediction of SAP [14]. C-reactive protein has the advantages of low cost and simple assay. Nevertheless, American College of Gastroenterology guidelines state that the utilization of C-reactive protein to predict severity in patients in AP is not practical as it takes $72 \mathrm{~h}$ to become accurate [7]. Mounzer et al. [3] suggested that the best classifiers in predicting the development of persistent organ failure at admission and $48 \mathrm{~h}$ after admission were modified Glasgow and JSS score, respectively. However, the AUC of modified Glasgow and JSS score was inferior 
to SABP and BISAP score in our study (Figs. 1, 5). This difference may be partly explained by the fact that we ruled out patients that had already developed organ failure at data collection, e.g. patients with $\mathrm{PaO}_{2}<60 \mathrm{mmHg}$ (respiratory failure). Which may result in a decrease of the total calculated score since $\mathrm{PaO}_{2}<60 \mathrm{mmHg}$ (respiratory failure) is one of the items included in both the Glasgow and JSS score [3]. The other possible explanation may be that these scoring systems were used as continuous variables in our study while they were converted into binary values in the study by Mounzer et al. [3] when calculating AUC. Dichotomization of a continuous predictor has many disadvantages, such as loss of information, reduction in power and increase in the probability of false positive results [30, 49].

The novelties and strengths of our study include the following: (i) To the best of our knowledge, this is the first study attempting to develop an index score using SAP defined by the up-to-date revised Atlanta criteria as the primary outcome. In addition, this is the first study to evaluate SIRS and pleural effusion as potential predictors of SAP defined by the up-to-date revised Atlanta criteria; (ii) Patients with acute pancreatitis could be divided into three groups according to different SABP scores according to CART analysis (Fig. 3), which is easy to use for risk stratification of acute pancreatitis at the bedside; (iii) SABP uses findings of vital signs, routine laboratory data, and imaging to derive a four-point score, which make it of similar simplicity to BISAP yet maintains a higher diagnostic accuracy [12]. The calculation of the modified Glasgow (eight points) and JSS score (nine points) is more complicated and these scores contain data not routinely collected at the time of hospitalization (e.g. lactate dehydrogenase, base excess, etc.); (iv)The SABP has another advantage over the Glasgow score in that it is calculated within $24 \mathrm{~h}$ of admission. American Gastroenterological Association Institute Guidelines propose that initial management decisions in AP can alter the course of disease and duration of hospitalization [50]. One of the expert's opinions is that the first $24 \mathrm{~h}$ ("golden hours") of care of patients with AP is crucial to reducing the morbidity and mortality. The modified Glasgow score require $48 \mathrm{~h}$ to complete, missing a potentially valuable early therapeutic window [12]; (v) The last advantage over other scoring systems is that SABP score could be used at different onset-to-admission times. AP is a dynamic and evolving process that involves multiple systems and the risk for organ complications [51]. We computed predicted probability of SAP over the SABP score from 0 to 10 for different onset-to-admission time (Fig. 4).

However, our study also has several limitations: Firstly, there were missing data for APACHE II, Glasgow score, JSS score and CRP in the derivation cohort due to retrospective study design, which may produce selection bias. However, the AUCs of these scores or markers were still lower than our SABP score when analysed in prospectively collected validation cohort with completed data. Secondly, we did not evaluate other putative risk factors, such as abdominal pressure and serum calcium despite the large number of candidate predictors which were examined. Lastly, radiologic scoring systems (such as computed tomography severity index, extrapancreatic score) have not been compared though many scoring systems were evaluated in our study. It will be interesting to compare our SABP score with such radiologic scoring systems in the future.

\section{Conclusions}

In conclusion, SIRS and pleural effusion are useful predictors of SAP defined by the up-to-date revised Atlanta criteria. SABP score might be a useful tool to stratify patients at risk of developing SAP defined by the up-todate revised Atlanta criteria and the application of it on admission may improve clinical care and management strategies in acute pancreatitis.

\section{Additional file}

Additional file 1: Table S1. Univariable analysis of predictive factors of severe acute pancreatitis in derivation.

\section{Abbreviations}

ALT: alanine aminotransferase; AP: acute pancreatitis; AST: aspartate aminotransferase; AUC: area under the curve of the receiver operating characteristic curve; BISAP: bedside index of severity in acute pancreatitis; BMI: body mass index; BUN: blood urea nitrogen; Cl: confidence interval; CT: computed tomography; ERCP: endoscopic retrograde cholangiopancreatography; HAPS: harmless acute pancreatitis score; IQR: interquartile range; OR: odds ratio; POF: persistent organ failure; PT: prothrombin time; ROC curve: receiver operating characteristic curve; SABP: systemic inflammatory response syndrome, albumin, blood urea nitrogen and pleural effusion; SAP: severe acute pancreatitis; SIRS: systemic inflammatory response syndrome; SD: standard deviation.

\section{Acknowledgements}

We thank Prof. Francois G. Briveta (a French retired previously University Hospital ICU department Head Director) for his invaluable comments on this manuscript.

\section{Authors' contributions}

WH: Study concept and design, acquisition of data, analysis and interpretation of data, drafting of the manuscript, critical revision and final approval of the manuscript. KDL: Critical revision, study supervision, and final approval of the manuscript. SP: Acquisition of data, critical revision and final approval of the manuscript. VZ: Critical revision, study supervision, and final approval of the manuscript. EK: Critical revision, study supervision, and final approval of the manuscript. SS: Critical revision and final approval of the manuscript. MZ: Critical revision and final approval of the manuscript. CW: Acquisition of data, critical revision and final approval of the manuscript. MZ: Analysis and interpretation of data; Critical revision and final approval of the manuscript. All authors read and approved the final manuscript.

Funding

None. 


\section{Availability of data and materials}

The datasets used and/or analysed during the current study are available from the corresponding author on reasonable request.

\section{Ethics approval and consent to participate}

This study protocol was approved by the Ethic Committee of the First Affiliated Hospital of Wenzhou Medical University. This study was performed according to the principles expressed in the Declaration of Helsinki and informed consent was obtained from the subjects.

\section{Consent for publication}

Not applicable.

\section{Competing interests}

The authors declare that they have no competing interests.

\section{Author details}

${ }^{1}$ Department of Gastroenterology and Hepatology, The First Affiliated Hospital of Wenzhou Medical University, Nanbaixiang, Ouhai District, Wenzhou 325000, Zhejiang, People's Republic of China. ${ }^{2}$ Department of Surgery, Massachusetts General Hospital Harvard Medical School, Boston, MA 02114, United States. ${ }^{3}$ Department of Gastroenterology and Hepatology, The First Affiliated Hospital of Wenzhou Medical University, Wenzhou, Zhejiang, People's Republic of China. ${ }^{4}$ Department of Medicine II, Saarland University Medical Center, Saarland University, 66424 Homburg, Germany. ${ }^{5}$ Department of Medicine, Marienhausklinik St. Josef Kohlhof, 66539 Neunkirchen, Germany. ${ }^{6}$ Division of Informatics, Imaging and Data Science, Faculty of Biology, Medicine and Health, University of Manchester, Manchester M13 9GB, UK. ${ }^{7}$ NIHR School for Primary Care Research, Centre for Primary Care and Health Services Research, University of Manchester, Manchester, UK. ${ }^{8}$ Department of Surgery, World Mate Emergency Hospital, Battambang, Cambodia. ${ }^{9}$ Unit of Gastroenterology and Digestive Endoscopy, Sandro Pertini Hospital, Rome, Italy. ${ }^{10}$ Department of Gastroenterology, The First Affiliated Hospital of Soochow University, Jiangsu, People's Republic of China. ${ }^{11}$ Department of Surgery, Key Laboratory of Diagnosis and Treatment of Severe Hepato-Pancreatic Diseases of Zhejiang Province, The First Affiliated Hospital of Wenzhou Medical University, Wenzhou, Zhejiang, People's Republic of China.

Received: 28 February 2019 Accepted: 2 May 2019

Published online: 08 May 2019

\section{References}

1. Hong W, Geng W, Chen B, Basharat Z, Wu Q, Zimmer V, Zhou M. Predictors of acute pancreatitis with low elevation of serum amylase. Ther Clin Risk Manag. 2017;13:1577-84.

2. Lankisch PG, Apte M, Banks PA. Acute pancreatitis. Lancet. 2015;386(9988):85-96.

3. Mounzer R, Langmead CJ, Wu BU, Evans AC, Bishehsari F, Muddana V, Singh VK, Slivka A, Whitcomb DC, Yadav D, et al. Comparison of existing clinical scoring systems to predict persistent organ failure in patients with acute pancreatitis. Gastroenterology. 2012;142(7):1476-82 (quiz e1415-e1476)

4. Raraty MG, Connor S, Criddle DN, Sutton R, Neoptolemos JP. Acute pancreatitis and organ failure: pathophysiology, natural history, and management strategies. Curr Gastroenterol Rep. 2004;6(2):99-103.

5. Hong W, Dong L, Huang Q, Wu W, Wu J, Wang Y. Prediction of severe acute pancreatitis using classification and regression tree analysis. Dig Dis Sci. 2011;56(12):3664-71.

6. Banks PA, Bollen TL, Dervenis C, Gooszen HG, Johnson CD, Sarr MG, Tsiotos GG, Vege SS. Classification of acute pancreatitis - 2012: revision of the Atlanta classification and definitions by international consensus. Gut. 2013;62(1):102-11.

7. Tenner S, Baillie J, DeWitt J, Vege SS, American College of G. American College of Gastroenterology guideline: management of acute pancreatitis. Am J Gastroenterol. 2013;108(9):1400-15.

8. Gardner TB, Vege SS, Pearson RK, Chari ST. Fluid resuscitation in acute pancreatitis. Clin Gastroenterol Hepatol. 2008;6(10):1070-6.

9. Warndorf MG, Kurtzman JT, Bartel MJ, Cox M, Mackenzie T, Robinson S, Burchard PR, Gordon SR, Gardner TB. Early fluid resuscitation reduces morbidity among patients with acute pancreatitis. Clin Gastroenterol Hepatol. 2011;9(8):705-9.

10. van Santvoort HC, Besselink MG, de Vries AC, Boermeester MA, Fischer K, Bollen TL, Cirkel GA, Schaapherder AF, Nieuwenhuijs VB, van Goor H, et al. Early endoscopic retrograde cholangiopancreatography in predicted severe acute biliary pancreatitis: a prospective multicenter study. Ann Surg. 2009;250(1):68-75.

11. Singla A, Simons J, Li Y, Csikesz NG, Ng SC, Tseng JF, Shah SA. Admission volume determines outcome for patients with acute pancreatitis. Gastroenterology. 2009;137(6):1995-2001.

12. Wu BU, Johannes RS, Sun X, Tabak Y, Conwell DL, Banks PA. The early prediction of mortality in acute pancreatitis: a large population-based study. Gut. 2008;57(12):1698-703.

13. Ueda T, Takeyama Y, Yasuda T, Kamei K, Satoi S, Sawa H, Shinzeki M, Ku Y, Kuroda Y, Ohyanagi H. Utility of the new Japanese severity score and indications for special therapies in acute pancreatitis. J Gastroenterol. 2009:44(5):453-9.

14. Lankisch PG, Weber-Dany B, Hebel K, Maisonneuve P, Lowenfels AB. The harmless acute pancreatitis score: a clinical algorithm for rapid initial stratification of nonsevere disease. Clin Gastroenterol Hepatol. 2009;7(6):702-5 (quiz 607).

15. Koutroumpakis E, Wu BU, Bakker OJ, Dudekula A, Singh VK, Besselink MG, Yadav D, van Santvoort HC, Whitcomb DC, Gooszen HG, et al. Admission hematocrit and rise in blood urea nitrogen at $24 \mathrm{~h}$ outperform other laboratory markers in predicting persistent organ failure and pancreatic necrosis in acute pancreatitis: a post hoc analysis of three large prospective databases. Am J Gastroenterol. 2015;110(12):1707-16.

16. Hong W, Lin S, Zippi M, Geng W, Stock S, Basharat Z, Cheng B, Pan J, Zhou M. Serum albumin is independently associated with persistent organ failure in acute pancreatitis. Can J Gastroenterol Hepatol. 2017;2017:5297143.

17. Royston P: PTREND: Stata module for trend analysis for proportions. 2014.

18. Cuzick J. A Wilcoxon-type test for trend. Stat Med. 1985;4(1):87-90.

19. de Madaria E, Banks PA, Moya-Hoyo N, Wu BU, Rey-Riveiro M, AcevedoPiedra NG, Martinez J, Lluis F, Sanchez-Paya J, Singh VK. Early factors associated with fluid sequestration and outcomes of patients with acute pancreatitis. Clin Gastroenterol Hepatol. 2014;12(6):997-1002.

20. Osada J, Wereszczynska-Siemiatkowska U, Dabrowski A, Dabrowska MI. Platelet activation in acute pancreatitis. Pancreas. 2012;41(8):1319-24.

21. Kakafika A, Papadopoulos V, Mimidis K, Mikhailidis DP. Coagulation, platelets, and acute pancreatitis. Pancreas. 2007;34(1):15-20.

22. Mentula P, Kylanpaa ML, Kemppainen E, Jansson SE, Sarna S, Puolakkainen $\mathrm{P}$, Haapiainen R, Repo H. Early prediction of organ failure by combined markers in patients with acute pancreatitis. Br J Surg. 2005;92(1):68-75.

23. Nelson JA, Chung CU, Fischer JP, Kanchwala SK, Serletti JM, Wu LC. Wound healing complications after autologous breast reconstruction: a model to predict risk. J Plast Reconstr Aesthet Surg. 2015;68(4):531-9.

24. Vilar-Gomez E, Yasells-Garcia A, Martinez-Perez Y, Calzadilla-Bertot L, Torres-Gonzalez A, Gra-Oramas B, Gonzalez-Fabian L, Villa-Jimenez O, Friedman SL, Diago M, et al. Development and validation of a noninvasive prediction model for nonalcoholic steatohepatitis resolution after lifestyle intervention. Hepatology. 2016;63(6):1875-87.

25. Thabane M, Simunovic M, Akhtar-Danesh N, Marshall JK. Development and validation of a risk score for post-infectious irritable bowel syndrome. Am J Gastroenterol. 2009;104(9):2267-74.

26. Sullivan LM, Massaro JM, D'Agostino RB Sr. Presentation of multivariate data for clinical use: the Framingham Study risk score functions. Stat Med. 2004;23(10):1631-60

27. Sachs MC. PlotROC: a tool for plotting ROC curves. J Stat Softw. 2017;79:C02.

28. Hong WD, Zhu QH, Huang ZM, Chen XR, Jiang ZC, Xu SH, Jin K. Predictors of esophageal varices in patients with HBV-related cirrhosis: a retrospective study. BMC Gastroenterol. 2009;9:11.

29. LeDell E, Petersen M, van der Laan M. Computationally efficient confidence intervals for cross-validated area under the ROC curve estimates. Electron J Stat. 2015;9(1):1583-607.

30. Steyerberg EW. Clinical prediction models: a practical approach to development, validation, and updating. New York: Springer; 2009. 
31. Meffert PJ, Baumeister SE, Lerch MM, Mayerle J, Kratzer W, Volzke H. Development, external validation, and comparative assessment of a new diagnostic score for hepatic steatosis. Am J Gastroenterol. 2014;109(9):1404-14.

32. Phillip V, Schuster T, Hagemes F, Lorenz S, Matheis U, Preinfalk S, Lippl F, Saugel B, Schmid RM, Huber W. Time period from onset of pain to hospital admission and patients' awareness in acute pancreatitis. Pancreas. 2013;42(4):647-54.

33. Gatta A, Verardo A, Bolognesi M. Hypoalbuminemia. Intern Emerg Med. 2012;7(Suppl 3):S193-9.

34. Kumar A, Chari ST, Vege SS. Can the time course of systemic inflammatory response syndrome score predict future organ failure in acute pancreatitis? Pancreas. 2014;43(7):1101-5.

35. Mofidi R, Duff MD, Wigmore SJ, Madhavan KK, Garden OJ, Parks RW. Association between early systemic inflammatory response, severity of multiorgan dysfunction and death in acute pancreatitis. Br J Surg. 2006;93(6):738-44.

36. Singh VK, Wu BU, Bollen TL, Repas K, Maurer R, Mortele KJ, Banks PA. Early systemic inflammatory response syndrome is associated with severe acute pancreatitis. Clin Gastroenterol Hepatol. 2009:7(11):1247-51.

37. Horwich TB, Kalantar-Zadeh K, MacLellan RW, Fonarow GC. Albumin levels predict survival in patients with systolic heart failure. Am Heart J. 2008;155(5):883-9.

38. Xue P, Huang ZW, Li YH, Guo J, Wang ZC, Zhao JL, You Z. Clinical study on severe acute pancreatitis associated with hypoalbuminemia in early stage. Zhong Xi Yi Jie He Xue Bao. 2005;3(6):443-5.

39. Hong W, Lin S, Zippi M, Geng W, Stock S, Zimmer V, Xu C, Zhou M. High-density lipoprotein cholesterol, blood urea nitrogen, and serum creatinine can predict severe acute pancreatitis. Biomed Res Int. 2017;2017:1648385.

40. Wu BU, Bakker OJ, Papachristou GI, Besselink MG, Repas K, van Santvoort HC, Muddana V, Singh VK, Whitcomb DC, Gooszen HG, et al. Blood urea nitrogen in the early assessment of acute pancreatitis: an international validation study. Arch Intern Med. 2011;171(7):669-76.

41. Maringhini A, Ciambra M, Patti R, Randazzo MA, Dardanoni G, Mancuso L, Termini A, Pagliaro L. Ascites, pleural, and pericardial effusions in acute pancreatitis: a prospective study of incidence, natural history, and prognostic role. Dig Dis Sci. 1996:41(5):848-52.
42. Heller SJ, Noordhoek E, Tenner SM, Ramagopal V, Abramowitz M, Hughes $M$, Banks PA. Pleural effusion as a predictor of severity in acute pancreatitis. Pancreas. 1997;15(3):222-5.

43. Caironi P, Tognoni G, Gattinoni L. Albumin replacement in severe sepsis or septic shock. N Engl J Med. 2014;371(1):84.

44. Salerno F, Navickis RJ, Wilkes MM. Albumin treatment regimen for type hepatorenal syndrome: a dose-response meta-analysis. BMC Gastroenterol. 2015;15:167

45. Salerno F, Navickis RJ, Wilkes MM. Albumin infusion improves outcomes of patients with spontaneous bacterial peritonitis: a meta-analysis of randomized trials. Clin Gastroenterol Hepatol. 2013;11(2):123-30.

46. Wilson PG, Manji M, Neoptolemos JP. Acute pancreatitis as a model of sepsis. J Antimicrob Chemother. 1998;41(Suppl A):51-63.

47. Singh VK, Wu BU, Bollen TL, Repas K, Maurer R, Johannes RS, Mortele KJ, Conwell DL, Banks PA. A prospective evaluation of the bedside index for severity in acute pancreatitis score in assessing mortality and intermediate markers of severity in acute pancreatitis. Am J Gastroenterol. 2009;104(4):966-71.

48. Muddana V, Whitcomb DC, Khalid A, Slivka A, Papachristou Gl. Elevated serum creatinine as a marker of pancreatic necrosis in acute pancreatitis. Am J Gastroenterol. 2009;104(1):164-70.

49. Royston P, Altman DG, Sauerbrei W. Dichotomizing continuous predictors in multiple regression: a bad idea. Stat Med. 2006;25(1):127-41.

50. Crockett SD, Wani S, Gardner TB, Falck-Ytter Y, Barkun AN. American gastroenterological association institute guideline on initial management of acute pancreatitis. Gastroenterology. 2018;154(4):1096-101.

51. Papachristou Gl, Muddana V, Yadav D, O'Connell M, Sanders MK, Slivka A, Whitcomb DC. Comparison of BISAP, Ranson's, APACHE-II, and CTSI scores in predicting organ failure, complications, and mortality in acute pancreatitis. Am J Gastroenterol. 2010;105(2):435-41 (quiz 442).

\section{Publisher's Note}

Springer Nature remains neutral with regard to jurisdictional claims in published maps and institutional affiliations.
Ready to submit your research? Choose BMC and benefit from:

- fast, convenient online submission

- thorough peer review by experienced researchers in your field

- rapid publication on acceptance

- support for research data, including large and complex data types

- gold Open Access which fosters wider collaboration and increased citations

- maximum visibility for your research: over 100M website views per year

At BMC, research is always in progress.

Learn more biomedcentral.com/submissions 